\title{
THE 2003 ISL RICH TRANSCRIPTION SYSTEM FOR CONVERSATIONAL TELEPHONY SPEECH
}

\author{
Hagen Soltau, Hua Yu, Florian Metze, Christian Fügen, Qin Jin, Szu-Chen Jou \\ Interactive Systems Laboratories \\ University of Karlsruhe (Germany), Carnegie Mellon University (USA) \\ \{soltau,metze,fuegen\}@ira.uka.de, \{hyu,qjin,scjou\}@cs.cmu.edu
}

\begin{abstract}
This paper describes the ISL large vocabulary conversational telephony speech recognition system, which was tested in NIST's RT-03S ("Switchboard") evaluation. We present our experiments on improving preprocessing, acoustic modelling, and language modelling. The system features phone-dependent semi-tied full covariances, semi-tied clustering of septa-phones, clustering across phones, feature adaptive training, robust estimation of VTLN and MLLR, as well as context-dependent interpolation of language models. We present detailed results for each stage of our multi-pass transcription scheme. System development started with a 1997 SWB system, yielding a word error rate of $35.1 \%$ on our internal $1 \mathrm{~h}$ development set. The final system performed at $21.8 \%$, a $38 \%$ relative improvement. The error rate on the RT-03 CTS evaluation set is $23.4 \%$.
\end{abstract}

\section{INTRODUCTION}

Recognition of conversational telephony speech is a challenging task, with respect to both acoustic and language modelling. Under-articulated speech causes a mismatch between pronunciation dictionary and acoustic models. Additionally, sloppy speech makes it hard to train appropriate language models. Furthermore, in 2003, automatic segmentation was required for the first time in the HUB- 5 evaluation series.

We started by reviving ISL's 1997 SWB system [1]. This multi-pass system obtained a top rank in the 1997 evaluation. Running this system as-is on the 2001 evaluation set, we achieved an error rate of $34.8 \%$. This system is significantly behind the top systems in the 2001 SWB evaluation [5], demonstrating that the ASR community achieved substantial improvements over the last years.

We used two test sets (table 1) for development purposes. Dev01 is a $1 \mathrm{~h}$ subset of Eval01, designed to have a similar error rate as the full set (35.1\% vs. 34.8\%). Tests with automatic segmentation were performed on the "Dryrun" data, which is a sub-set of Eval02. Unless otherwise stated, the reported error rates are based on Dev01. Since several setups for different experiments were used during system development, the stated error rates do not decrease monotonously and the results need to be viewed with respect to the corresponding base-line.

The paper is organised as follows: First, we present experiments for acoustic modelling including front-end and segmentation. Next, we describe our language models and the decoding strategy and present results for each system stage. In the final section, we describe experiments to reduce the decoding time.

\begin{tabular}{|l|c|c|}
\hline & subset from & segmentation \\
\hline \hline Dev01 & 1h from Eval01 & manual \\
\hline Dry-run & 1h from Eval02 & automatic \\
\hline
\end{tabular}

Table 1: Development sets.

\section{ACOUSTIC MODELLING}

Acoustic models were trained by merging three corpora: $265 \mathrm{~h}$ of SWB and Callhome, $32 \mathrm{~h}$ of cellphone, and $65 \mathrm{~h}$ of "CTRAN" SWB-2 data. The cellphone and CTRAN data were weighted by a factor of 3 and 2 , respectively. The original ISIP training transcripts were used for the SWB data. The training dictionary was derived from CMUdict and on average contains nearly 2 pronunciation variants per base-form.

Since we started a new training environment, we performed several steps to clean-up the data-base. By discarding all training segments containing one word only, an error reduction from $37.1 \%$ to $36.4 \%$ was obtained. Furthermore, we limited the segment boundaries to max. 15 frames of silence only. Zero-energy frames observed in parts of some conversations lead to extreme likelihoods, in particularly in combination with feature space adaptation. Discarding these frames by using a zero-crossing feature resulted in an improvement from $33.4 \%$ to $32.8 \%$. Additionally, segments with poor likelihoods were removed as well.

\subsection{Preprocessing}

The $5 \mathrm{~min}$ excerpts from the conversations were segmented into smaller chunks before decoding. The segmentation works in two phases. An initial energy-based segmentation with three categories (speech, non-speech, unsure) is used to bootstrap GMMs for speech and non-speech. These GMMs are used to re-segment the unsure parts. Finally, a smoothing process is conducted to join adjacent chunks.

As shown in table 2, segmentation error rate and word error rate are not necessarily correlated. The best automatic segmentation is only $0.8 \%$ worse then manual segmentation. 


\begin{tabular}{|l|c|c|}
\hline system & seg. error (NIST score) & WER \\
\hline \hline manual & - & $41.1 \%$ \\
\hline automatic & $14.3 \%$ & $41.9 \%$ \\
\hline automatic & $9.7 \%$ & $43.0 \%$ \\
\hline
\end{tabular}

Table 2: Segmentation on Dry-run.

The front-end is based on 13 mel-filtered cepstral coefficients per frame, applying conversation side wide cepstral mean subtraction. Incorporating context information by concatenating 11 frames gave significantly better results than a $\Delta$-based approach. The final feature vectors are transformed by an LDA using the context dependent states as classes and the dimension is reduced from 143 to 42 .

\begin{tabular}{|l|c|}
\hline front-end & WER \\
\hline \hline$\Delta$ 's+ $\Delta \Delta$ 's+LDA & $39.7 \%$ \\
\hline frame stacking+LDA & $38.5 \%$ \\
\hline+ CVN & $37.6 \%$ \\
\hline
\end{tabular}

Table 3: Front-end improvements.

Our VTLN estimation procedure maximises the likelihood for voiced sounds. Traditionally, warping factors were estimated with fixed CMS/CVN which introduces inconsistencies. Our revised iterative estimation of all front-end parameters computes the likelihood for a given warping factor with the correct CMS/CVN vectors. This makes it desirable to use a more efficient search method than line search. The new procedure is, therefore, based on Brent search. The interleaved estimation yielded an improvement from $33.2 \%$ to $32.4 \%$.

\subsection{Training procedure}

The training procedure is based on fixed state alignments. In our experiment, the alignments were generated with a small context dependent system. As shown in table 4, these labels are significantly better than the labels generated with the full setup ${ }^{1}$. We attribute this result to the better generalisation capability. Moreover, this approach outperforms both viterbi and forward/ backward training. Generating a set of frame/ state alignments once and keeping it fixed over several training iterations reduces the training time drastically compared to traditional training. This will become an important issue once 2000h of Fisher training data become available.

Our traditional training procedure bootstrapped the models with the K-means algorithm. As an alternative, we implemented an "incremental growing of Gaussians"procedure. Starting with one component per state, the Gaussians will be splitted along the largest covariances. An occupancy threshold is used to deactivate "dead" Gaussians. The training consists of 7 big iterations with parameter doubling. After each big iteration, three "small"

\footnotetext{
${ }^{1}$ The number of parameters is $7 \%$ of the number of parameters in the full setup.
}

\begin{tabular}{|l|c|}
\hline alignment & WER \\
\hline \hline full fwd/bwd & $33.1 \%$ \\
\hline viterbi & $33.3 \%$ \\
\hline labels with full setup & $33.5 \%$ \\
\hline labels with small setup & $32.7 \%$ \\
\hline
\end{tabular}

Table 4: Forced alignments.

re-estimation steps are performed without splitting. This strategy is particularly advantageous for the $10^{\prime} 000 \times 32$ setup (see table 5, where the models consist of $10^{\prime} 000$ states with 32 Gaussians. The final models have $288^{\prime} 000$ Gaussians due to the integrated pruning. Combining the "incremental growing" strategy with fixed alignments leads to a very time and memory efficient training, as the preprocessed data can now be organised per context-dependent HMM state. Therefore, the training can be parallelised according to the states instead of the dialogues as usual, drastically reducing file-IO.

\begin{tabular}{|l|c|c|}
\hline method & 10'000x24 & 10 '000x32 \\
\hline \hline init with k-means & $33.8 \%$ & $33.7 \%$ \\
\hline incremental growing & $33.1 \%$ & $32.4 \%$ \\
\hline
\end{tabular}

Table 5: Training procedure.

\subsection{Clustering}

Context dependent models are created by an Entropy-based clustering procedure. First, mixture weights for all polyphone models are trained on top of context independent codebooks. Questions about the phonetic context and the phone position are used to split the tree nodes. Extending the context from \pm 2 to \pm 3 yields a gain from $34.7 \%$ to $34.2 \%$. The clustering is applied in two stages: in the first stage, a tree with $10 k$ leafs is generated for the full model parameters. In a second stage, $50 k$ states are grown on the leafs of the first tree for the mixture weights only. The extended tree has $5 \%$ extra model parameters, but reduces the word error rate to $31.8 \%$ (from $32.8 \%$, see table 6 ).

\begin{tabular}{|l|c|c|}
\hline acoustic models & \# params & WER \\
\hline 10k CBs $+10 \mathrm{k}$ DSs & $27.2 \mathrm{M}$ & $32.8 \%$ \\
\hline 10k CBs $+50 \mathrm{k}$ DSs & $28.5 \mathrm{M}$ & $31.8 \%$ \\
\hline
\end{tabular}

Table 6: Two-level Clustering.

Traditional clustering grows one tree per context independent HMM state. As an alternative, we investigated across-phone trees [10], offering better parameter sharing capabilities. This clustering procedure grows 6 trees only ("begin", "middle", and "end" for vowels and consonants) and implicitly modeling articulatory changes in sloppy speech. 


\begin{tabular}{|l|c|c|c|}
\hline Clustering & dict. & train $(66 \mathrm{~h})$ & train $(180 \mathrm{~h})$ \\
\hline \hline traditional & multiple & $34.4 \%$ & $33.4 \%$ \\
\hline across phones & multiple & $33.9 \%$ & - \\
\hline traditional & single & $34.1 \%$ & - \\
\hline across phones & single & $33.1 \%$ & $31.6 \%$ \\
\hline
\end{tabular}

Table 7: Clustering across Phones. Note the behaviour on dictionaries containing single or multiple pronunciation variants.

\subsection{Semi-tied full covariances}

Semi-tied full covariances (STC) [3] attempt to reduce the detrimental effects of diagonal covariance modelling. The STC parameters are trained on top of the LDA transform. Our estimation procedure estimates all parameters, e.g. diagonal covariances and STC transforms, simultaneously, resulting in a significant memory footprint. However, the containers for the statistics can be allocated on demand. In combination with the parallelisation over the HMM states, the memory footprint can be divided by the number of parallelised jobs. As shown in table 8, phone dependent STC classes do not work in combination with MLLR. Therefore, phone dependent STC classes are used only for the first, unadapted, decoding pass.

\begin{tabular}{|l||c|c|}
\hline & w/o MLLR & with MLLR \\
\hline \hline no STC & $36.7 \%$ & $34.1 \%$ \\
\hline global STC & $33.7 \%$ & $32.2 \%$ \\
\hline phone STC & $33.4 \%$ & $33.1 \%$ \\
\hline
\end{tabular}

Table 8: Interaction of STC with MLLR.

STC training is also applied for the test speakers using the recogniser output. The global STC classes are re-estimated for each test speaker (in addition to MLLR and FSA, see below) and results in a minor improvement $(26.8 \% \rightarrow 26.6 \%)$.

\subsection{Feature Space Adaptation (FSA)}

Feature space adaptation is used both in training and testing. The adaptive training is carried out per conversation side on top of the LDA/STC transforms. The VTLN factors are kept fixed during FSA re-estimation. A determinant constraint $|A|=1$ is induced during the matrix estimation in contrast to constrained MLLR [2]. As shown in table 9, FSA gives a $1.1 \%$ improvement on top of all other normalisation and adaptation techniques. In contrast to FSA, where only one global matrix is used, MLLR makes use of a regression tree and the number of transforms depends on the adaptation data available.

\subsection{MMIE training}

The accumulation strategy for discriminative training is based on confusion networks [4]. First, lattices are generated using an uni-gram language model (LM). A down-

\begin{tabular}{|l||c|}
\hline setup & WER \\
\hline \hline VTLN,MLLR,STC & $28.9 \%$ \\
\hline + FSA-SAT & $27.8 \%$ \\
\hline
\end{tabular}

Table 9: Feature Space Adaptation.

scaling of the LM scores is applied to "flatten" the word posterioris. Next, lattices are converted to confusion networks. The Forward/Backward procedure is applied to these networks. The word boundaries can therefore be adjusted during training in contrast to the "phone-marked lattice"approach [9]. However, both accumulation procedures lead to the same results. A weighted ML and MMIE criterion is used to update the parameters. Only one iteration is used; the second iteration already led to over-training on the full setup. The discriminative training leads to an error reduction from $28.3 \%$ to $27.6 \%$ on the full setup (LDA, VTLN, STC, FSA-SAT, MLLR, two-level clustering).

\begin{tabular}{|l||c|c|}
\hline setup & ML & MMIE \\
\hline \hline small & $41.9 \%$ & $40.9 \%$ \\
\hline full & $28.3 \%$ & $27.6 \%$ \\
\hline
\end{tabular}

Table 10: Discriminative Training.

\section{LANGUAGE MODELLING}

The search vocabulary contains $41 \mathrm{k}$ base-forms and $96 \mathrm{k}$ pronunciations selected from SWB, BN, and CNN corpora. The pronunciations were either taken from CMUdict or generated by Festival. Pronunciation probabilities were treated as penalties during decoding and as real probabilities for confusion network generation. The frequencies were generated from training labels. Three separate LMs were interpolated, using predecessor-dependant weights. As shown in table 11, the CNN LM did not improve the performance. Thus, the combined LM consists of 3gram SWB +5 gram class SWB + 4gram BN. Apart from the first, unadapted, pass, all passes used the full interpolated LM during decoding and lattice generation.

\begin{tabular}{|l|l|}
\hline 3gram SWB & $31.4 \%$ \\
\hline+ 5gram class SWB & $31.0 \%$ \\
\hline+ 4gram BN & $30.3 \%$ \\
\hline+ 4gram CNN & $30.5 \%$ \\
\hline
\end{tabular}

Table 11: Language Modelling, context dependent interpolation.

\section{DECODING}

The search engine is a one-pass decoder based on linguistic polymorphisms [6]. The full LM history is conserved in lin- 


\begin{tabular}{|l||l|l|}
\hline Pass 0 & $35.0 \%$ & Tree-150, MMIE, STC-50, smallLM \\
\hline Pass 1/2 & $28.5 \%$ & Tree-150, ML, STC-1, VTLN, MLLR, bigLM \\
\hline Pass 3/4 & $27.2 \%$ & Tree-150, MMIE, STC-1, VTLN, MLLR, FSA-SAT, bigLM \\
\hline Pass 5 & $26.6 \%$ & Tree-6, ML, STC-1, FSA-SAT, bigLM, SPDict \\
\hline Pass 6 & $26.2 \%$ & Tree-150, MMIE, STC-1, VTLN, MLLR, FSA-SAT, bigLM \\
\hline Pass 7 & $26.4 \%$ & Tree-6, cross-adaptation \\
\hline Pass 8 & $25.4 \%$ & Tree-150, cross-adaptation \\
\hline Pass 9 & $24.7 \%$ & System Combination \\
\hline
\end{tabular}

Table 12: Decoding Passes (Tree-150= clustering per phone state, Tree- $6=$ clustering across phones, STC-50= phone dependent classes, STC-1= global STC), results on Dry-run (automatic segmentation).

guistic instances of search nodes. Subgraph dominance is therefore exploited implicitely. The search network is based on a general graph structure, sharing roots and tails. Isomorphic subgraphs are merged via an iterative procedure reducing redundancies. Lattice nodes are created from the active search space by removing the LM information. Links are created during and after decoding. This allows to transfer as much information as possible from the active search space into the lattice.

The decoding passes are summarised in table 12. Pass 0 used phone dependent STC classes and the 3gram SWB LM only. Lattice based MLLR [8] is used to generate adapted models for the next pass. Passes 7 and 8 are used for cross-adaptation between the Tree-150 (traditional clustering) and Tree- 6 (clustering across phones) setup. The effect of cross-adaption can be seen by comparing passes 6 and 8 which use the same models. System combination uses a mixture of Rover and confusion network combination. We fuse lattices from different stages into one single confusion network. Overall, processing of the test data took about 190 times real-time on a $2.4 \mathrm{GHz}$ Pentium 4 single CPU.

Since fast transcription systems receive increasing interest, we investigated the trade-off between speed and accuracy on the final, adapted, decoding pass. The final decoding pass with the adapted models runs in 12 times realtime with open search beams and gives $24.2 \%$ WER on the Eval03 test set. As shown in table 13, the decoding process can operate in real time with a moderate increase of search errors if appropriate beam settings are used.

\begin{tabular}{|l||c|c|}
\hline Pruning parameter & RTF & WER \\
\hline beam $=2.4$ (eval mode) & 12.0 & 24.2 \\
\hline beam $=1.5$ & 4.7 & 24.6 \\
\hline beam $=1.1$ & 1.4 & 26.0 \\
\hline + transN $=35$ & 1.0 & 26.1 \\
\hline + delayed LM interpolation & 0.9 & 26.1 \\
\hline
\end{tabular}

Table 13: Single adapted pass, RTF on P4 2.4Ghz, WER on Eval03.

\section{SUMMARY}

We described the development of ISL's 2003 transcription system for conversational telephony speech. The sys- tem achieved an error rate of $23.4 \%$ on the official RT-03 (Eval03) CTS test set. Starting last year with a WER of $35.1 \%$ on our dev01 development set, improvements of acoustic and language modelling led to a WER of $21.8 \%$.

Part of this work has been funded by the European Union as IST project No. IST-2000-28323 (FAME).

\section{REFERENCES}

[1] M. Finke, J. Fritsch, P. Geutner, K. Ries, and T. Zeppenfeld. The JanusRTk Switchboarch/Callhome 1997 Evaluation System. In LVCSR Hub-5E Workshop, Linthicum Heights, MD, USA, 1997.

[2] M.J.F. Gales. Maximum likelihood linear transformations for HMM-based speech recognition. Technical report, Cambridge University, England, 1997.

[3] M.J.F. Gales. Semi-Tied Covariance Matrices for Hidden Markov Models. IEEE Transactions on Speech and Audio Processing, Vol. 2, May 1999.

[4] L. Mangu, E. Brill, and A. Stolcke. Finding Consensus among words: Lattice-based word error minimization. In Proceedings of the Eurospeech, Hungary, 1999.

[5] A. Martin and M. Przybocki. The 2001 NIST Evaluation for Recognition of Conversational Speech Over the Telephone. In LVCSR Workshop, Linthicum Heights, MD, USA, 2001.

[6] H. Soltau, F. Metze, C. Fügen, and A. Waibel. A One Pass Decoder based on polymorphic linguistic context assignment. In Proceedings of the Automatic Speech and Recognition Workshop (ASRU), Trento, Italy, 2001.

[7] H. Soltau, T. Schaaf, F. Metze, and A. Waibel. The ISL Evaluation System for Verbmobil-II. In Proceedings of the ICASSP, Salt Lake City, USA, 2001.

[8] L. Uebel and P. Woodland. Improvements in linear transform based speaker adaptation. In Proceedings of the ICASSP, Salt Lake City, USA, 2001.

[9] P. Woodland and D. Povey. Large Scale Discriminative Training of Hidden Markov Models forh Speech Recognition. Computer Speech and Language, 6, 2002.

[10] Hua Yu and Alex Waibel. Flexible Parameter Tying for Conversational Speech Recognition. In Proceedings of SSPR, Tokyo, Japan, 2003. 